\title{
Building Fire Evacuation model research Based on an Improved Fruit Fly Optimization Algorithm
}

\author{
Lijie Zhang ${ }^{1,2, a}$, Jianchang Liu' ${ }^{1, b}$, Shubin Tan ${ }^{1, c}, X i a ~ Y u^{1, d}$ \\ ${ }^{1}$ School of Information Science and Engineering, Northeastern University Shenyang,110006 China \\ ${ }^{2}$ Experiment and Practice Centre Ningxia Institute of Science and Technology Shizuishan, 753000 \\ China
}

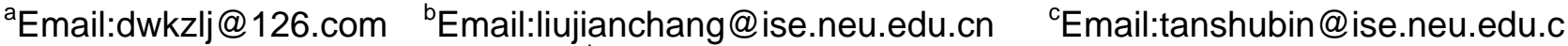
n dEmail:yuxia@ise.neu.edu.cn

Keywords: Emergency Routing; Evacuation Times; IFOA; Flow Rate

\begin{abstract}
We take into account to find a set of rapid evacuation routes during a building fire. We construct a three-dimensional building structure of the network, an evacuation route is a sequence of movements of people away from the threat of fire, to a safe exit in the network. The fire can stress crowds, cause their competitive behaviors, lead to disorder and blocking as they pass through the nearest passages and exits. This paper provide a novel improvement fruit fly (IFOA) algorithm for the 3D route of evacuation with different node flow rate limiting characterizing. The algorithm has been proved feasible and effective after test the database.
\end{abstract}

\section{Introduction}

In recent years, there is a growing concern over the problem of fire safety evacuation [1]. Because high-rise building has a fire spread fast, it is very difficult to evacuation and rescue. How to make the staff out of fire in the finite evacuation time, that is the important content of the building safety design.

During the last decade, the crowd evacuation modeling of research findings have the queuing model[2], continuous crowd flow [3]. Typical models are building EXODUS[4]; EXITT[5]; SIMULEX[6]. This model can not reflects the individual characteristics, interaction between individuals in disasters. For the shortest routes choose, Cova et al[7] propose a lane-based large-scale evacuation network flow model. Campos[8] provide a k-shortest path algorithm. Chen[9] provide a monophyletic fastest flow algorithm. Andreas [10] use LP and nonlinear programming. It can not reflects the influence of structure and environment on the crowd evacuation behaviors and mentality.

In this paper, we construct a three-dimensional building structure of the evacuation network, an evacuation route is a sequence of movements of people away from the threat of fire, to a safe exit in the network. The sensory of fire can stress crowds, lead to disorder and blocking as they pass through the nearest passages and exits, the further stairways and exits can be not make full use. How to optimize crowd guidance is an important problem. We provide a novel improvement fruit fly (IFOA) algorithm for the 3D path of evacuation with different node flow rate limiting. By dispersing passages capacity and evading the nearest exits, to avoid possible disorder and blocking. This work is further the extension the former work. We stress considered practical problem two main requirements: (i) The method should be improved the defects of conventional evacuation guidance. It is very fast to be used by the evacuation supervisors in real time. (ii) It should be provide effective help to be not familiar with the environment of evacuees.

We apply a graphical presentation for modelling building evacuation.The graph is the three first floors of the laboratory building on the Northeastern University. Experiments are conducted to verify the feasibility and effectiveness of our proposed approach.

The rest of the paper is organized as follows: In the next section we introduce network model of building space. In Section 3 we present the principles of basic FOA and improved FOA. In Sections 4 we apply the model to solve evacuation problem, and the experiment result analysis. We provides 
conclusions and future work in Section 5.

\section{Network Model of Building Space}

\section{Properties of Building Evacuation Space}

Network nodes and evacuation passages propertie are defined and described as follow. The structural of system is represented as $G=(W, E)$ a network, where each area is denoted by a vertex $w \in W$ and each passage connecting areas is denoted by an arc $e \in E$. Nodes set $W=\left\{w_{1}, w_{2}, \ldots, w_{n}\right\}$, and definition $w_{i}\left(I D_{i}, x_{i}, y_{i}, z_{i}, t, k_{i}(t), n_{i}(t)\right)$, where $I D_{i}$ is the ith node number, $x_{i}, y_{i}, z_{i}$ building space the three-dimensional coordinates, respectively, $k_{i}(t)$ is the integrated comprehensive information of fire in anytime $t, n_{i}(t)$ is the number of evacuee from the source node in anytime $t$. The edges correspond different passageways and stairs. Passage set $E=\left\{w_{1} w_{2}, w_{2} w_{3}, \ldots, w_{i} w_{j}, \ldots, w_{m} w_{n}\right\}$.

\section{A Three-Dimensional Path Planning Problem Description}

Path planning is to find the shortest sequence from the source nodes to the exit. The definitions are as follows:

The definition 1: any point in floor $w_{i}\left(x_{i}, y_{i}, z_{i}\right)$, if the edge $e_{w_{i} w_{j}}$ isn't intersect with any obstacles, to store the set $W_{\text {allowde }}\left(x_{i}, y_{i}, z_{i}\right)$.

The definition 2: the dist anceany two adjacent node $w_{i}, w_{j}, L_{w_{i} w_{j}}$ are shown in:

$$
L_{w_{i} w_{j}}=\sqrt{\left(x_{i}-x_{j}\right)^{2}+\left(y_{i}-y_{j}\right)^{2}+\left(z_{i}-z_{j}\right)^{2}}
$$

By definition 2 we can be calculated out the distance between any two nodes.

Considering the diffusion of fire, the evacuation route $d_{w_{i} w_{j}}$ calculation formula:

$$
d_{w_{i} w_{j}}=k l_{w_{i} w_{j}}
$$

where $k$ is the integrated comprehensive information fire.

\section{Modeling Nomenclature}

$N$ total number of evacuees. $R_{l}$ evacuees to choose the route, $l=1,2, \ldots, L$

$n_{i l}$ the number of people through the path $r_{l}$ and node $w_{i} . R C_{i}$ the maximum flow rate of passage $R_{l}, \quad R C_{l}=\min \left\{c_{i j} \mid e_{i j} \in R_{l}\right\}, c_{i j}=n_{i l}(t) / l_{i j}$

$C_{i}^{U B}$ the maximum crowd floe rate of node the unit time, person $/ \mathrm{m} \cdot \mathrm{s}$

$T_{r_{l}}$ the time for route $r_{l}, T_{r_{l}}=\sum_{e_{i j} \in r_{l}} \frac{d_{i j}}{v}, v$ the flat straight road walking speed

$T_{w_{i}}$ the time for a group evacuees to go through the exit $T_{w_{i}}=\left\{\begin{array}{l}\frac{n_{i l}}{R C_{l}}, R C_{l} \leq C_{i}^{U B} \\ \frac{n_{i}}{C_{i}^{U B}}, R C_{l}>C_{i}^{U B}\end{array}\right.$

$T_{i l}$ total evacuation time any node and path, $T_{i l}=T_{r_{1}}+\max T_{w_{i}}$, depending on the number of people through the node into the path

The objective function and constraints :

$$
\begin{gathered}
\min f_{1}=\sum_{i=1}^{K} \sum_{l=1}^{L} T_{i l}\left(n_{i l}\right) \\
\text { s.t. } \sum_{i=1}^{K} \sum_{l=1}^{L} n_{i l}(t)=N \\
\frac{n_{i l}(t)}{C_{i}^{U} \cdot t} \leq 1
\end{gathered}
$$




\section{Improve FOA Algorithm(IFOA)}

\section{The Basic FOA and Applying}

The Fruit Fly Optimization Algorithm (FOA) was proposed by Pan[10]. FOA is a new swarm intelligence method based on the food finding behavior of the fruit fly[11]. The olfactory organ of fruit flies can discover the floating scents in the air. Then use its sensitive vision to find food and fly towards that direction [12].

Since FOA has been proposed, FOA has been applied in several applications, such as neural network parameters controller optimization[13,14], and the knapsack problem etc.[15].

IFOA for the Model

Consider to devise the fitness functions is defined as:

$f(X)=\min \left\{d\left(X, w_{j}\right), w_{j} \in W_{\text {allowed }}\right\}$

IFOA is divided into several necessary steps, The steps are:

Step 1. Initialization.Given the maximum Maxgen $K_{\max }$ and sizepop. Set the lower bound $\left(C^{L B}\right)$ and the upper bound $\left(C^{U B}\right)$ of $C_{i}$.sub-swarms number $M$.Initialize fruit fly swarm location Init $X_{-}$axis $, j=1,2, \ldots, n$ which represents each node data variable, $i=1,2, \ldots$, popsize which shows each individual fruit flies, $m=1,2, \ldots, M$ which represents each sub-swarm.

Step 2. Randomized initial fruit fly swarm location matrix:

$X_{i, j, m}=X_{-}$axis $_{m}+R(k) * \operatorname{rand}[0,1]$

with

$R(k)=\left(\frac{C_{i}^{U B}-C_{i}^{L B}}{2}\right)\left(\frac{k \max -k}{k \max }\right)^{\phi}$

where $\phi=2-6$. rand $\in[0,1]$.

Step 3: The distance of food source to the initialization location is calculated as follows:

Dist $_{i j}=X_{i j}$

Dist $_{i}$ should be limited by the following rule:

Dist $_{i j}=\left\{\begin{array}{l}C_{i}^{U B}, \text { Dist }_{i j}>C_{i}^{U B} \\ C_{i}^{L B}, \text { Dist }_{i j}<C_{i}^{U B}\end{array}\right.$

Step 4. Find the fitness value ( $\left(\mathrm{smell}_{i}\right)$ of the individual location of fruit fly.

Step 5. Find out the fruit fly with the minimum value among each sub-swarm.

Step 6. Judge and update the fitness value of each sub-swarm.

Step7. Update the global fitness and best position $X_{-}$axis among multi-swarm.

Step 8. Perform cooperative local search method by

$X_{-}$new $=\frac{1}{M} \sum_{m=1}^{M} X_{-}$axis $_{m}$

Step 9. Vision searching process. the probability of fruit flies choosing the next exit $P\left(w_{j}\right)$ is:

$P\left(w_{j}\right)=\left\{\begin{array}{cc}\frac{\varpi_{1} x(j)+\varpi_{2} x_{-} n e w(j)}{\sum_{j \in w_{\text {allowde }}\left(\varpi_{1} x(j)+\varpi_{2} x \_n e w(j)\right)}, j \in\{\text { reachable neighbors }\}} \\ 0 \quad, j \in\{\text { unreachable neighbors }\}\end{array}\right.$

where $\varpi_{1}$ and $\varpi_{2}$ are weights, and $\varpi_{1}, \varpi_{2} \in(0,1)$.

Step 10. If $k \geq$ Maxgen, stop the IFOA search, otherwise, go to Step 2 .

\section{Experimental Result}

The model and IFOA algorithm were used MATLAB7.0 coding and run on the PC with $2.53 \mathrm{GHz}$, and $2 \mathrm{G}$ memory. The initial population is 20 and 30 , muti swarm number $3, \sigma_{1}=0.4$, and the iteration $k=200,300,500.1000$. Fig. 1 fruit fly flying route, Fig. 2 (a) (b)The best fitness based on 
IFOA and FOA, respectively. Further analyze the performance of the FOA and IFOA in 3D evacuation space. The average evacuation time computed by two algorithms for different number of people are drawn in Fig. 3. we are compared 200, 300, 500, 800 people evacuation time. The results show that all the people are transferred to safe exit region by time period 480 s. The results demonstrate that the proposed model is fit for solving buildings fire evacuation problem.

We consider a graph fragment earlier presented. The evacuation routes are depicted in Fig.4.This optimum route consists of 10 nodes connection as follows: 1-7-3-11-9-15-16-20-18-21. People select different route towards the exit as time goes on. Therefore, congestion on the nearest exits is avoided.

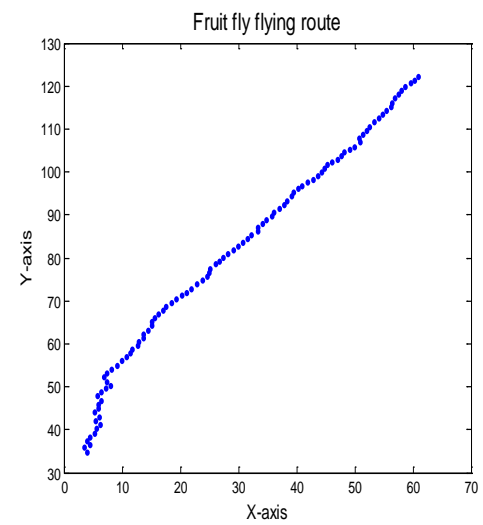

Fig. 1 fruit fly flying route
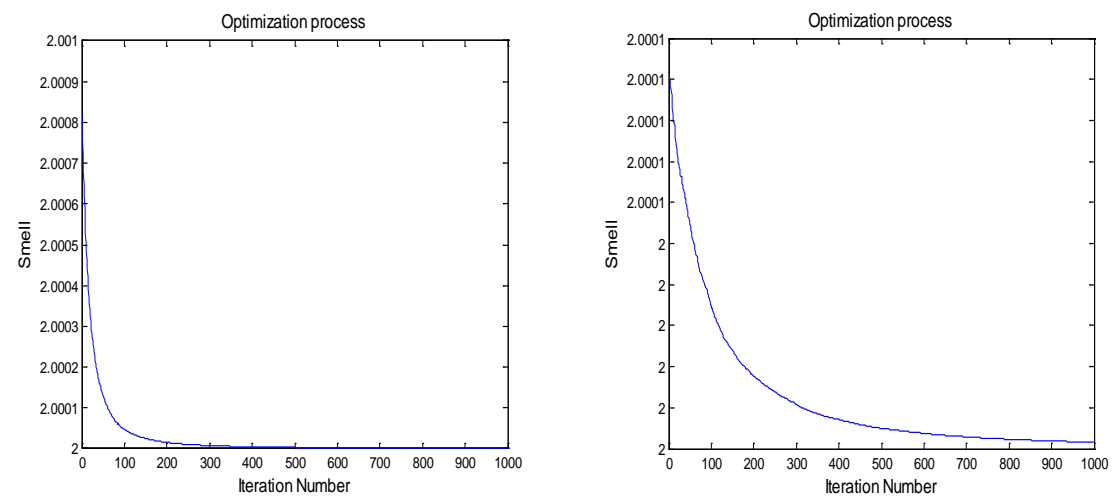

Fig. 2 (a) The best fitness based on IFOA Fig. 2 (b) The best fitness based on FOA

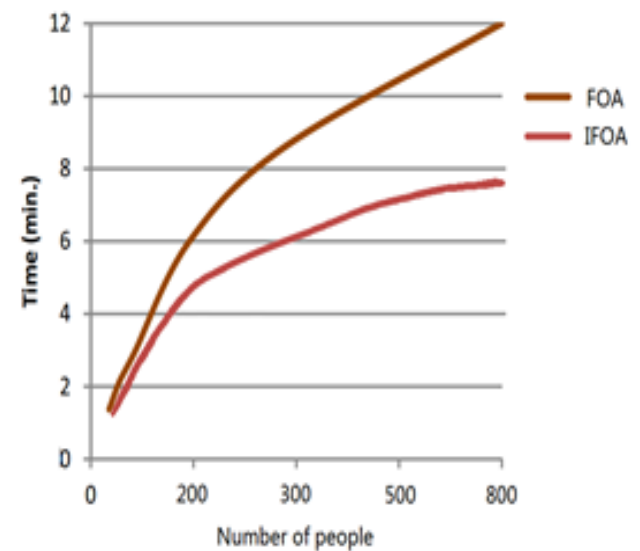

Fig. 3. The average evacuation time for different number of people

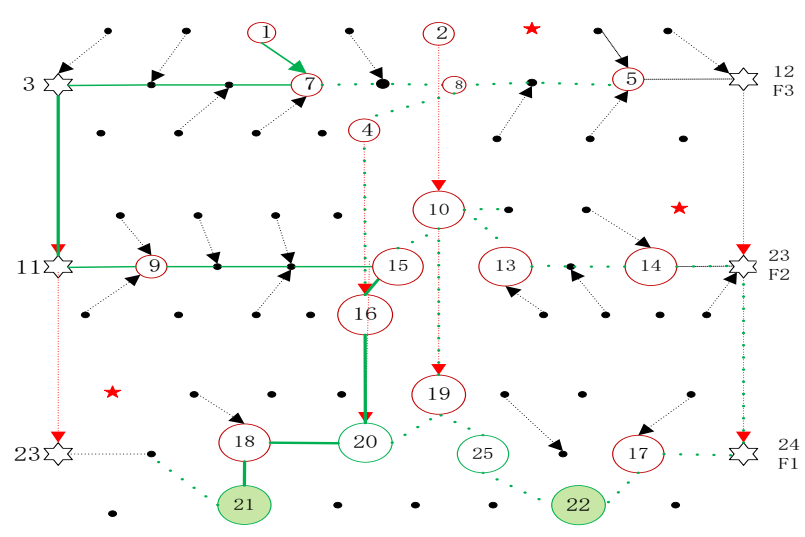

Fig. 4. The optimum evacuation routes in the building

\section{Conclusion and Future Work}

This paper propose a new macroscopic swarm intelligence model where fire, smoke, and individual factors can guide a crowd to rationl escape. By dispersing passages capacity, to avoid 
possible disorder and blocking. The algorithm has been proved feasible and effective after test the database.

In future work, we consider to study the use of adaptive evacuee routing techniques that can help better evacuate. Future work on swarm intelligence algorithms will also consider a realistic distribution of users in the building.

\section{Acknowledgment}

This work was partially supported by the National Natural Science Foundation of China, under Grants 61374137, and the State Key Laboratory of Integrated Automation of Process Industry Technology and Research Center of National Metallurgical Automation Fundamental Research Funds, under Grant 2013ZCX02-03.

\section{References}

[1] YanWenhua, KongXiangkun, HaoAiling, QiuPeifang. Developments of human behavior research in fires of China [J].Fire Science and Technology,2012, 31(11):1225-1228(04):702-709.

[2] Yamada, T., 1996. A network of approach to a city emergency evacuation planning, International Journal of Systems Science 27(10), p. 931.

[3] Owen, M., Galea, E. R., Lawrence, P. J., 1997. “Advanced occupant behavioural features of the building EXODUS evacuation model,” Proceedingsof the fifth international symposium, pp. 795.

[4] Kostreva, M. M., Lancaster, L. C., 2000. A comparison of two methodologies in Hazard I fire egress analysis, Fire Technology 34(3), p. 227.

[5] Lo, S. M., Fang, Z., Lin, P., et al., 2004. An Evacuation Model: the SGEM Package, Fire Safety Journal 39(3), p.169.

[6] Cova, T. J., Johnson, J. P., 2003. A network flow model for lane-based evacuation routing, Transportation Research Part A 37,p.579.

[7] Campos V, Da Silva P, Evacuation transportation planning:A method of identify optimal independent routes [C]. proceedings of Urban Transport V: Urban Transport and the Environment for the 21st Century. Southampton:WIT Press,2000:555-564.

[8] Chen PH,Feng F. A fast flow control algorithm for real-time emergency evacuation in large indoor areas [J]. Fire Safety Journal, 2009,44(5):732-740

[9] Desmet, A., \& Gelenbe, E. (2013). Graph and analytical models for emergency evacuation. Future Internet, 5, 46 - 55.

[10] W.T. Pan, A new fruit fly Optimization algorithm: taking the financial distress model as an example, Knowledge Based Syst. 26 (2012) 69 - 74.

[11] W.T. Pan, Using modified fruit fly optimisation algorithm to perform the function test and case studies, Connection Sci. 25 (2 - 3) (2013) 151 - 160.

[12] R. Storn, K. Price, Differential evolution - a simple and efficient heuristic for global optimization over continuous spaces, J. Global Optim. 11 (1997)341 - 359.

[13] W.T. Pan, A new fruit fly Optimization algorithm: taking the financial distress model as an example, Knowledge Based Syst. 26 (2012) 69 - 74.

[14] H. Li, S. Guo, C. Li, J. Sun, A hybrid annual power load forecasting model based on generalized regression neural network with fruit fly optimizationalgorithm, Knowledge Based Syst. 37 (2013) 378 - 387.

[15] L. Wang, Y.L. Shi , S. Liu. An improved fruit fly optimization algorithm and its application to joint replenishment problems.Expert Systems with Applications.Expert Systems with Applications 42 (2015) 4310 - 4323. 